\title{
Neural progenitor cells from human induced pluripotent stem cells generated less autogenous immune response
}

\author{
HUANG Ke ${ }^{1 \dagger}$, LIU PengFei ${ }^{1,2 \dagger}$, LI Xiang ${ }^{1}$, CHEN ShuBin ${ }^{1}$, WANG LiHui ${ }^{1}$, QIN Li ${ }^{3}$, \\ SU ZhengHui ${ }^{1}$, HUANG WenHao ${ }^{1}$, LIU JuLi ${ }^{1}$, JIA Bei $^{4}$, LIU Jie ${ }^{4}$, CAI JingLei ${ }^{1 *}$, \\ PEI DuanQing ${ }^{1 *} \&$ PAN GuangJin $^{1 *}$ \\ ${ }^{1}$ Key Laboratory of Regenerative Biology, Guangdong Provincial Key Laboratory of Stem Cell and Regenerative Medicine, Guangzhou \\ Institutes of Biomedicine and Health, Chinese Academy of Sciences, Guangzhou 510530, China; \\ ${ }^{2}$ Department of Regeneration Medicine, School of Pharmaceutical Science, Jilin University, Changchun 130021, China; \\ ${ }^{3}$ Laboratory of Pathogen Biology, State Key Laboratory of Respiratory Disease, Center for Infection and Immunity, Guangzhou Institutes of \\ Biomedicine and Health, Chinese Academy of Sciences, Guangzhou 510530, China; \\ ${ }^{4}$ Department of Obstetrics and Gynecology, Nanfang Hospital, Southern Medical University, Guangzhou 510515, China
}

Received August 17, 2013; accepted September 20, 2013; published online January 16, 2014

\begin{abstract}
The breakthrough development of induced pluripotent stem cells (iPSCs) raises the prospect of patient-specific treatment for many diseases through the replacement of affected cells. However, whether iPSC-derived functional cell lineages generate a deleterious immune response upon auto-transplantation remains unclear. In this study, we differentiated five human iPSC lines from skin fibroblasts and urine cells into neural progenitor cells (NPCs) and analyzed their immunogenicity. Through co-culture with autogenous peripheral blood mononuclear cells (PBMCs), we showed that both somatic cells and iPSC-derived NPCs do not stimulate significant autogenous PBMC proliferation. However, a significant immune reaction was detected when these cells were co-cultured with allogenous PBMCs. Furthermore, no significant expression of perforin or granzyme B was detected following stimulation of autogenous immune effector cells $\left(\mathrm{CD}^{+} \mathrm{CD}^{-} \mathrm{T}\right.$ cells, $\mathrm{CD}^{+} \mathrm{CD} 8^{+} \mathrm{T}$ cells or $\mathrm{CD}^{-} \mathrm{CD}^{-} 6^{+}$ NK cells) by NPCs in both PBMC and T cell co-culture systems. These results suggest that human iPSC-derived NPCs may not initiate an immune response in autogenous transplants, and thus set a base for further preclinical evaluation of human iPSCs.
\end{abstract}

induced pluripotent stem cells, immunogenicity, iPSC-derived neural progenitor cells

Citation: Huang K, Liu PF, Li X, Chen SB, Wang LH, Qin L, Su ZH, Huang WH, Liu JL, Jia B, Liu J, Cai JL, Pei DQ, Pan GJ. Neural progenitor cells from human induced pluripotent stem cells generated less autogenous immune response. Sci China Life Sci, 2014, 57: 162-170, doi: $10.1007 / \mathrm{s} 11427-013-4598-6$

The ability of human embryonic stem cells (ESCs) to differentiate into all three embryonic germ layers represents a great potential avenue for cell-based transplantation medicine $[1,2]$. Recently, therapeutics derived from human ESCs have been used in animal models of diseases, such as Par-

$\dagger$ Contributed equally to this work

*Corresponding author (email: pan_guangjin@gibh.ac.cn; pei_duanqing@gibh.ac.cn; cai_jinglei@gibh.ac.cn) kinson's disease [3,4], ischemic-reperfusion injury [5], and spinal cord injury [6]. However, several challenges to human ESC-based therapy have arisen. Aside from ethical concerns and a low efficacy in establishment, immune rejection of allogenous transplantation currently limits the serious application of ESCs in clinical studies. These issues have been resolved by the development of induced pluripotent stem cells (iPSCs). iPSCs are reprogrammed from so- 
matic cells by the use of four defined factors (Oct4, Sox2, $\mathrm{Klf} 4$, and c-Myc) $[7,8]$. iPSCs have similar characteristics to those of ESCs, including unlimited growth capacity, multi-lineage in vitro differentiation, germline transmission, and even contribution to create entire animals [9-11]. However, the overwhelming advantage of iPSCs technology over ESCs is that patient-specific iPSCs are easily generated [12-14]. Moreover, iPSC-derived functional cells have been reported to successfully treat animal models of sickle-cell anemia and Parkinson's disease, amongst others [15-18]. Thus, iPSC technology offers the unprecedented opportunity to use human cells to study human diseases with further application to clinical therapies free of the immune rejection.

Despite these promises, whether cells derived from iPSCs are tolerated by the immune system remains uncertain. Fairchild expressed doubts regarding the lack of immunogenicity of iPSCs and iPSC-derived cell types as early as 2010 [19]. Shortly thereafter, Zhao et al. [20] reported that the transplantation of undifferentiated mouse iPSCs induced a T-cell-dependent immune response even in a syngeneic mouse. In this study, teratomas formed by B6 iPSCs were mostly immune-rejected by B6 recipients, while B6 ESCs were not. Several genes, including $\mathrm{Zg} 16$ and Hormad1, were found to directly affect the immunogenicity of iPSC derivatives. However, undifferentiated iPSCs would never be used in a treatment application because of their ability to randomly differentiate into teratomas. Therefore, it is possible that T-cell infiltration appears in the developing teratomas [21]. Recently, Araki et al. [22] demonstrated a limited or absent immune response, including T-cell infiltration, to differentiated skin and bone marrow tissues derived from mouse iPSCs. Additionally, no significant difference in the rate of transplantation success of iPSC-derived versus ESC-derived tissues was identified. Moreover, expression of $\mathrm{Zg} 16$ and Hormad1 genes was not increased in regressing skin and teratoma tissues. However, in this study the differentiation process was performed in vivo, which varies from the course of differentiation in vitro. Thus, the model used in this study does not exactly represent the approach to clinical therapies using human iPSCs. More recently, Guha et al. [23] found no increase in $\mathrm{T}$ cell proliferation in vitro, no antigen-specific secondary immune response and no rejection of iPSC-derived embryoid bodies (EBs) and tissue-specific cells after their transplantation into syngeneic mice. They further observed little evidence of an immune response to undifferentiated syngeneic mouse iPSCs following transplantation. Therefore, they concluded that differentiated cells derived from syngeneic mouse iPSC transplants were not rejected. However, all of the above studies were performed in mouse models, and the immunogenicity of human iPSCs and iPSC derivatives has not yet been explored intensively.

In the current study, we investigated the immunogenicity of human iPSCs derived from adult skin fibroblasts (SFs) and urine cells (UCs). Following differentiation of iPSCs into neural precursor cells (NPCs), autogenous and allogenous immune responses to NPC stimulation were compared. NPCs derived from human iPSCs did not induce an obvious immune response from autogenous immune cells, while a significant response from allogenous immune cells was observed. These findings indicate that human iPSC derivatives could potentially be applied for auto-transplantation without inducing immune rejection.

\section{Materials and methods}

All samples were collected following principles approved by the Guangzhou Institute of Biomedicine and Health Ethical Committee. All volunteers who donated skin, urine or blood samples have signed consent forms upon request.

\subsection{Cell culture and iPSC generation}

Human adult skin fibroblasts (SFs) and urine cells (UCs) were obtained from healthy volunteers 20-40 years old. Human SFs were cultured in Dulbecco's Modified Eagle's Medium (DMEM; Hyclone, Logan City, Utah, USA) supplemented with penicillin/streptomycin (Hyclone), Lglutamine (Gibco, Carlsbad, California, USA), non-essential amino acids (NEAA; Gibco) and $10 \%$ fetal bovine serum (FBS; PAA, Queensland, Australia). Human UCs were cultured in primary medium containing DMEM/F12 1:1 (Hyclone), 10\% FBS, SingleQuot Kit CC-4127 Renal Epithelial Growth Medium (REGM; Lonza, Basel, Switzerland), amphotericin B and penicillin/streptomycin for $2 \mathrm{~d}$ and then changed to Renal Epithelial Basal Medium (Lonza) containing SingleQuot Kit CC-4127 REGM for subsequent days [24,25]. Both SF-derived iPSCs (SF-iPSCs) and UC-derived iPSCs (UC-iPSCs) were obtained from South Stem Cell Bank in Guangzhou, China. SF-iPSCs were generated by transduction using pMX-based retroviruses by supplementation with Vitamin $\mathrm{C}$ and valproic acid as described [26]. UC-iPSCs were induced by oriP/EBNA episomal vectors carrying a combination of the reprogramming factors OCT4, SOX2, SV40LT, KLF4 and miR302/367 through electroporation [27,28]. H1 human ESCs were purchased from WiCell (WA01/H1; WiCell, USA). Both H1 ESCs and other human iPSCs were cultured on matrigel (BD Biosciences, Franklin, New Jersey, USA) using mTeSR1 medium (Stemcell, Vancouver, Canada). For teratoma formation, three million hESCs or hiPSCs were injected subcutaneously into severe combined immunodeficiency mice. Teratomas were sectioned after eight weeks, and further examined for ZG16 and HORMAD1 expression.

\subsection{NPC differentiation in vitro}

H1 ESC and iPSC colonies were detached with $0.2 \mathrm{mg} \mathrm{mL}^{-1}$ 
Dispase (Gibco) and grown in suspension for $4 \mathrm{~d}$ in EB medium (DMEM/F12, 20\% knockout serum replacement (Gibco), NEAA, penicillin/streptomycin, L-glutamine and beta-mercaptoethanol (Gibco)) supplemented with $5 \mu \mathrm{mol}$ $\mathrm{L}^{-1}$ SB431542 (Calbiochem, Darmstadt, Germany) and 5 $\mu \mathrm{mol} \mathrm{L}{ }^{-1}$ dorsomorphin (Sigma, St. Louis, Missouri, USA). ESC- or iPSC-derived EBs were treated with neural induction medium containing DMEM/F12, N2 supplement (Gibco), heparin ( $2 \mu \mathrm{g} \mathrm{mL}^{-1}$, PeproTech, Crescent AveRocky Hill, USA), and NEAA for a further two days followed by adhesion to a matrigel-coated plate. After two weeks' culture, neural rosettes were blown off and cultured in suspension for a further 2-4 d in N2B27 medium (1:1 DMEM/F12 and Neurobasal medium (Gibco), N2 supplement, B27 supplement (Gibco), $20 \mathrm{ng} \mathrm{mL}^{-1}$ epidermal growth factor (PeproTech) and $10 \mathrm{ng} \mathrm{mL}^{-1}$ b-fibroblast growth factor (Sigma)). Neural spheres (suspensions of NPCs) were formed, purified by several passages, and harvested to examine the expression of neuronal markers NESTIN and PAX6 via flow cytometry and immunofluorescence staining.

\subsection{Isolation of PBMCs and $\mathrm{T}$ cells and proliferation assay}

Human peripheral blood mononuclear cells (PBMCs) were isolated from the blood of healthy volunteers with density-gradient centrifugation as described [29-32]. Briefly, OptiPrep $^{\mathrm{TM}}$ density gradient solution (Axis-shield, Oslo, Norway) was added to peripheral blood samples at a $1: 8$ ratio followed by centrifugation at $900 \times g$ for $30 \mathrm{~min}$. PBMCs were collected from the interphase and washed twice with RPMI 1640 medium (Gibco). T cells within PBMCs were isolated by incubation with CD3 MicroBeads (Miltenyi, Cologne, Germany) and separated using a MiniMACS ${ }^{\text {TM }}$ Separator with an MS Column (Miltenyi). Both PBMCs and T cells were maintained in RPMI 1640 medium containing $10 \%$ FBS. Cell viability was examined by trypan blue exclusion staining.

Co-culture experiments were carried out as described previously [30,33,34]. Culture volumes of $100 \mu \mathrm{L}$ per well in a 96-well plate were used, with the ratio of antigen cells to PBMCs kept at 1:100. Before co-culture, antigen cells were pre-treated with mitomycin $\mathrm{C}$. Detailed information for all groups is displayed in Table 1. Following a three-day co-culture, PBMC proliferation was examined using a cell proliferation BrdU ELISA kit (Roche Diagnostics, Basel, Switzerland) according to the manufacturer's instructions. The reaction was quantified by measuring the optical density at a wavelength of $370 \mathrm{~nm}$ with a reference wavelength of $492 \mathrm{~nm}$. The degree of proliferation was calculated as $\left(A_{370}-A_{492}\right)-\left(A^{\prime}{ }_{370}-A^{\prime}{ }_{492}\right)$, where $A=$ mean absorbance value of experimental group and $A^{\prime}=$ mean absorbance value of the blank group. Analysis was performed with Graphpad Prism 5.0. Three allogenous PBMC samples and one autogenous PBMC were used in the co-culture system. The final proliferation level of the negative group was regarded as one, and the proliferation level of experimental group was analyzed in relation to this.

\subsection{Assessment of cytotoxic molecule expression in the co-culture system}

$1 \times 10^{6}$ PBMCs or $\mathrm{T}$ cells were added to each well of a 96-well plate in a $200 \mu \mathrm{L}$ volume. The ratio of antigen cells to PBMCs/T cells was $1: 100$. The PBMC co-culture system had both positive and negative control groups, while the $\mathrm{T}$ cell co-culture system negative control group contained lymphocytes only and $\mathrm{T}$ cells in the positive control group were activated with Human T-Activator CD3/CD28 Dynabeads $^{\circledR}$ (Life Technologies, Carlsbad, California, USA) as per the manufacturer's instructions. Following overnight coculture, the expression levels of cytotoxic molecules (perforin and granzyme $\mathrm{B}$ ) in $\mathrm{CD} 3^{+} \mathrm{CD} 8^{-} \mathrm{T}$ cells, $\mathrm{CD} 3^{+} \mathrm{CD} 8^{+} \mathrm{T}$ cells and $\mathrm{CD}^{-} \mathrm{CD}^{-} 6^{+} \mathrm{NK}$ cells were examined by flow cytometry.

\subsection{Real-time quantitative PCR (qPCR)}

Total RNA was extracted with Trizol (Invitrogen, Carlsbad, California, USA) and reverse-transcribed using an RT-PCR kit (TaKaRa, Tokyo, Japan). qPCR was performed using a Thermal Cycler Dice ${ }^{\mathrm{TM}}$ Real Time System and SYBR Green Premix EX Taq ${ }^{\mathrm{TM}}$ (TaKaRa). Relative quantification of target gene expression was determined relative to that of $\beta$-actin and all measurements were in triplicate. Primer sequences $\left(5^{\prime} \rightarrow 3^{\prime}\right)$ were as follows: ZG16 (forward: TCCGG-

Table 1 Design of each group in the PBMC co-culture system ${ }^{\text {a) }}$

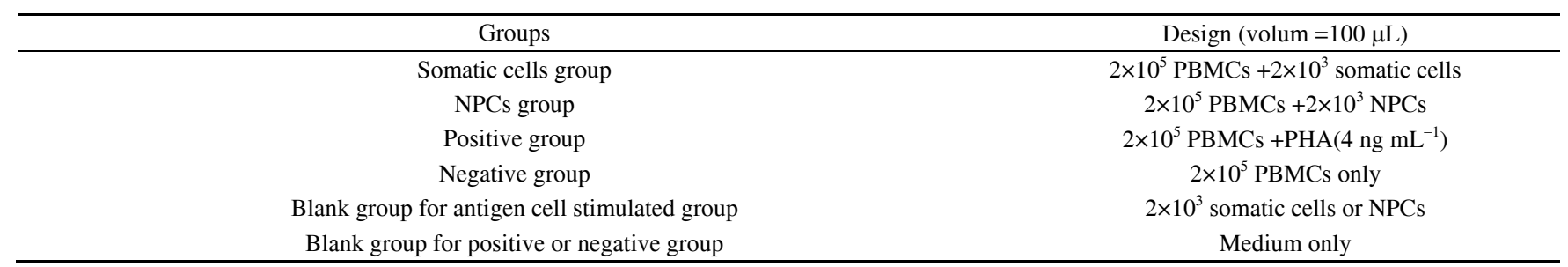

a) The ratio of antigen cells to PBMCs was kept at 1:100, and $100 \mu \mathrm{L}$ was set up as a volume system per well in a 96 -well plate. 
GTCCGAGTCAACA; reverse: TCCTCCAGGTCTCCGTTGC); HORMAD1 (forward: GCCCAGTTGCAGAGGACTC; reverse: TCTTGTTCCATAAGCGCATTCT); $\beta$ ACTIN (forward: CCCAGAGCAAGAGAGG; reverse: GTCCAGACGCAGGATG).

\subsection{Immunofluorescence staining}

Cells were fixed for 20 min in $4 \%$ paraformaldehyde dissolved in $0.1 \mathrm{~mol} \mathrm{~L}^{-1}$ phosphate buffer solution (PBS) and washed thrice with $0.01 \mathrm{~mol} \mathrm{~L}^{-1} \mathrm{PBS}$. Cells were further incubated with primary antibodies (Rabbit anti-NESTIN, 1:1000 (Millipore, Billerica, MA, USA); Mouse anti-PAX6, 1:1000 (Developmental Studies Hybridoma Bank, Lowa city, Lowa, USA)) in PBS with $10 \%$ goat serum and $0.3 \%$ Triton X-100 overnight at $4{ }^{\circ} \mathrm{C}$. Species-specific secondary antibodies conjugated to Alexa Fluor ${ }^{\circledR} 448$ or 568 (1:400, Invitrogen) were used to visualize primary antibodies. Nuclei were counterstained with 4',6-diamidino-2-phenylindole (DAPI; Sigma). Samples were mounted in anti-fade medium (Invitrogen) and preserved at $4^{\circ} \mathrm{C}$.

\subsection{Flow cytometry}

NPCs derived from iPSCs (SF-NPCs and UC-NPCs) were prepared at a concentration of $1 \times 10^{5}$ cells in $100 \mu \mathrm{L}$ PBS containing $2 \%$ FBS, while PBMCs and T lymphocytes were prepared at $1 \times 10^{6}$ cells in $100 \mu \mathrm{L}$ PBS containing $2 \%$ FBS. Antibodies used were NESTIN-PerCP-Cy ${ }^{\mathrm{TM}}$ 5.5, PAX6Alexa Fluor ${ }^{\circledR}$ 488, CD3-PercP, CD8-FITC, CD56-APC, perforin-PE and granzyme B-PE (all purchased from $\mathrm{BD}$ Biosciences). Antibodies were added and incubated for 30 min at $4^{\circ} \mathrm{C}$. After two washes in PBS, cells were acquired and analyzed on a FACSCalibur (BD Biosciences).

\subsection{Statistical analysis}

Values for qPCR and PBMC proliferation were expressed as mean \pm SEM. Differences between groups were analyzed by one-way analysis of variance (ANOVA) followed by $t$-tests. The $P$-value was set at 0.05 . All statistical analyses were performed with SPSS 17.0.

\section{Results}

\subsection{NPC differentiation from hESCs/hiPSCs}

NPC differentiation from hESCs and hiPSCs derived from both SFs and UCs was performed as described previously $[26,30,35,36]$. The typical neural rosettes were blown off and picked out to produce a neural sphere consisting of
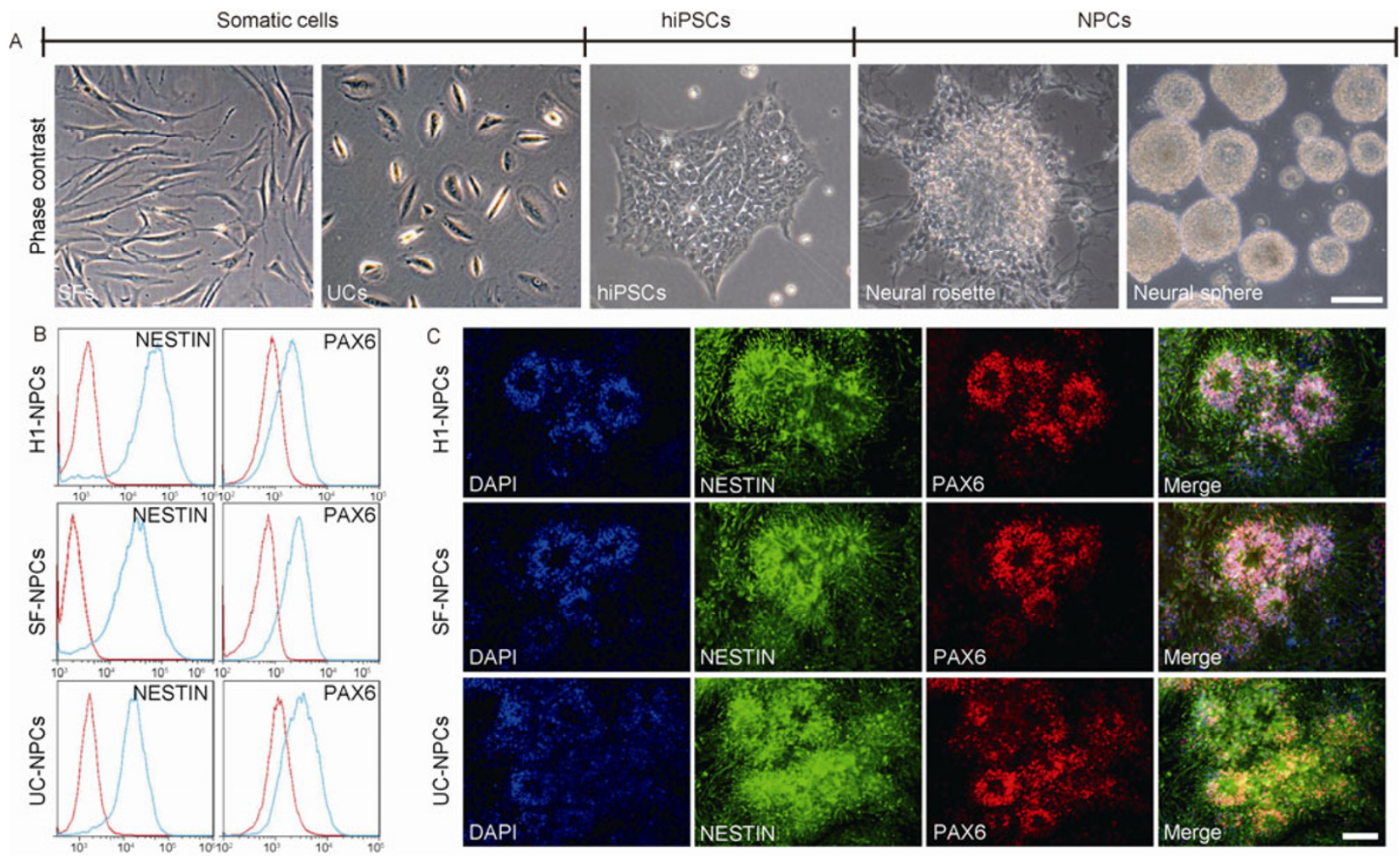

Figure 1 Characterization of human ESC/iPSC-derived NPCs. A, Phase contrast micrographs of somatic skin fibroblasts (SFs) and urine cells (UCs)), somatic cell-derived iPSCs, iPSC-derived neural rosettes and neural spheres. B, Flow cytometry analysis of neural markers (NESTIN and PAX6) in H1-ESC/iPSC-derived NPCs (H1-NPCs, SF-NPCs, UC-NPCs). C, Immunofluorescence microscopy depicting NESTIN (green) and PAX6 (red) in NPCs. DAPI is shown in blue. Scale bar, $100 \mu \mathrm{m}$. 
NPCs (Figure 1A). The differentiated cells from both cell origins were indeed NPCs confirmed with NPC markers (NESTIN and PAX6). Both SF-NPCs and UC-NPCs expressed the NPC marker NESTIN at a similar level to that detected in H1 hESC-derived NPCs (Figure 1B). The NPC marker PAX6 was also expressed in a similar manner from both cell types (Figure 1B). After attachment to culture plates, NPCs displayed the polarity typical of such cells. Positive expression of NESTIN (green) and PAX6 (red) was detected by immunofluorescence (Figure 1C). Therefore, the iPSC-derived NPCs used here retain the characteristics of NPC imprinting.

\subsection{PBMC proliferation in a co-culture system}

We next examined the immunogenicity of iPSC-derived NPCs. NPCs served as antigens and were co-cultured with autogenous or allogenous PBMCs. For the measurement of PBMC proliferation in each sample, the degree of prolifera- tion of PBMCs stimulated with NPCs was analyzed relative to a negative group with slight modification to the method previously described [33]. Reactions of the somatic cell lines (SF3, UC5) and the relative NPCs (SF3-NPCs, UC5NPCs) are displayed as examples (Figure 2A). No significant proliferation was observed when PBMCs were stimulated with either autogenous SF3 or SF3-NPCs (Figure 2A). Meanwhile, significant immune reactions were detected when SF3 or SF3-NPCs were co-cultured with three different allogenous PBMCs (Figure 2A). UC5 and UC5-NPC co-culture did not induce significant activation of autogenous PBMC proliferation, while obvious proliferation was observed in three different allogenous PBMC co-culture systems (Figure 2B). When compared with autogenous PBMCs, the relative proliferation level of each allogenous PBMC co-culture condition was significantly higher $(P<0.05)$. The relative proliferation levels stimulated by each cell line are summarized in Figure 2C. These data revealed that human iPSC-derived NPCs do not induce im-
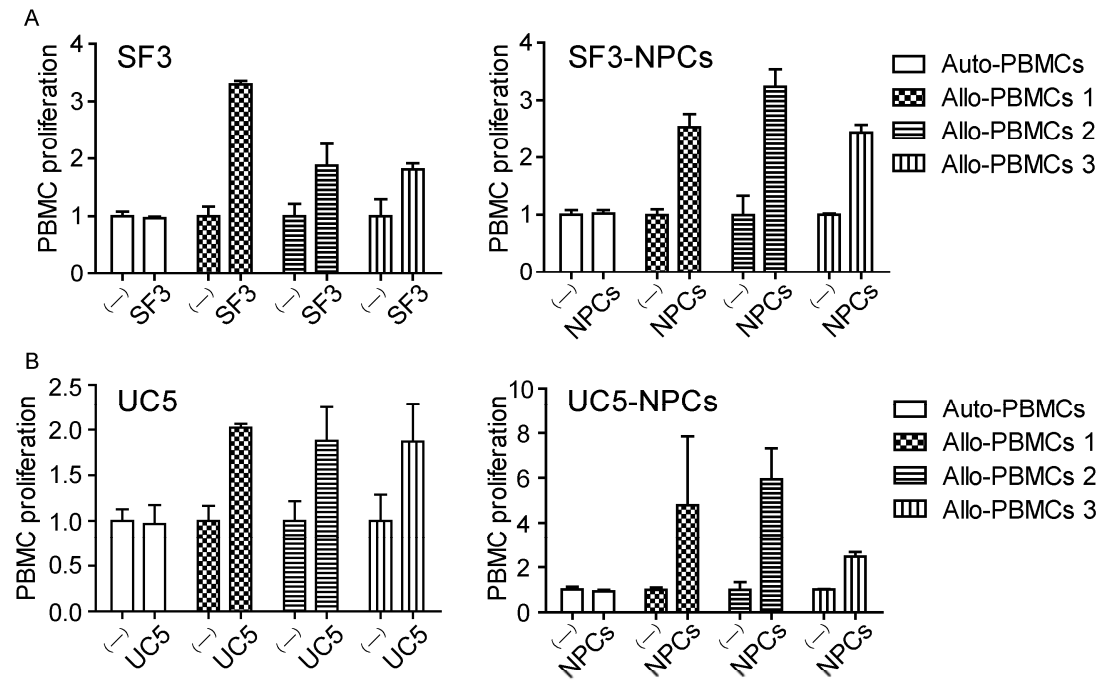

$\square$ Auto-PBMCs

Do. Allo-PBMCs 1

E Allo-PBMCs 2

mII Allo-PBMCs 3

\begin{tabular}{|c|c|c|c|c|c|}
\hline \multicolumn{2}{|c|}{ CELL TYPE } & Auto-PBMCs & Allo-PBMCs 1 & Allo-PBMCs 2 & Allo-PBMCs 3 \\
\hline \multirow{2}{*}{ SF1 } & Somatic cells & - & $* *$ & $*$ & - \\
\cline { 2 - 6 } & NPCs & - & $* *$ & $* *$ & $*$ \\
\hline \multirow{3}{*}{ SF3 } & Somatic cells & - & $* * *$ & $*$ & $*$ \\
\cline { 2 - 6 } & NPCs & - & $* *$ & $* * *$ & $* *$ \\
\hline \multirow{2}{*}{ UC2 } & Somatic cells & - & $* *$ & $* *$ & - \\
\cline { 2 - 7 } & NPCs & - & $* * *$ & $* * *$ & $* * *$ \\
\hline \multirow{3}{*}{ UC5 } & Somatic cells & - & $* *$ & $*$ & $*$ \\
\cline { 2 - 7 } & NPCs & - & $* * *$ & $* * *$ & $* *$ \\
\hline \multirow{2}{*}{ UC7 } & Somatic cells & - & $* *$ & $* * *$ & $* *$ \\
\cline { 2 - 6 } & NPCs & - & $* *$ & $* *$ & - \\
\hline
\end{tabular}

Figure 2 Proliferation of PBMCs stimulated by somatic cells and NPCs derived from SF and UC origins in a co-culture system. A, Relative PBMC proliferation in the co-culture system when stimulated with SF3 (left) and SF3-NPCs (right). B, Relative PBMC proliferation in the co-culture system when stimulated with UC5 (left) and UC5-NPCs (right). C, Summary of the relative autogenous or allogenous PBMC proliferation level following stimulation by somatic cells or NPCs from two SF and three UC donors in the co-culture experiment. Results are expressed as mean \pm SEM, and differences among groups were analyzed by one-way analysis of variance (ANOVA) followed by the single sample $t$-tests. "-" indicates a relative proliferation level of less than 1.5 when compared with the negative group; “*” indicates a relative proliferation level of $1.5-2$; “**” indicates a relative proliferation level of $2-3$; “***” indicates a relative proliferation level greater than 3 . 
mune response in autogenous cells.

\subsection{Distinct expression of the cytotoxic molecules per- forin and granzyme $B$ in co-culture system}

We further investigated whether the distinct kinetics profiles of perforin and granzyme B existed in autogenous PBMCs or T lymphocytes when co-cultured with iPSCderived NPCs. The percentage of perforin and granzyme B expression in $\mathrm{CD}^{+} \mathrm{CD}^{-} \mathrm{T}$ cells, $\mathrm{CD} 3^{+} \mathrm{CD} 8^{+} \mathrm{T}$ cells or $\mathrm{CD}^{-} \mathrm{CD}^{-} 6^{+} \mathrm{NK}$ cells was analyzed in PBMCs or $\mathrm{T}$ lymphocyte co-cultures with iPSC-derived NPCs via flow cytometry (Figures 3A and 4A). No activation of expression of perforin or granzyme B was detected in any of the cell groups within autogenous PBMC populations when stimulated by both NPC cell types (Figure 3B and C; Supplemental Files 1 and 2 in Supporting Information). However, significant activation of perforin expression by all three immune cell groups was observed in allogenous PBMCs following co-culture with both NPCs (Figure 3B and C).

Similar activation of perforin expression was detected in the T cell co-culture system (Figure 4B; Supplemental File 3 in Supporting Information). With the exception of the one allogenous $\mathrm{T}$ cell group stimulated by SF1-NPCs that showed a modest increase in relative expression of granzyme B, all other groups showed no significant activation, including autogenous T cell groups (Figure 4C; Supplemental File 4 in Supporting Information). The results also raised the consistent opinion that human iPSC-derived NPCs do not activate immune cells and initiate immune response in autogenous cells.

\section{Discussion}

iPSCs are considered potentially more useful than ESCs for studying and treating human diseases, with functional iPSC derivatives displaying promising progress towards clinical applications $[12,13,15,16]$. The immunogenicity of iPSC derivatives is one of the most important issues that needs to be addressed before clinical therapies using iPSC techniques can proceed $[19,20,22]$. Currently, this issue is high-
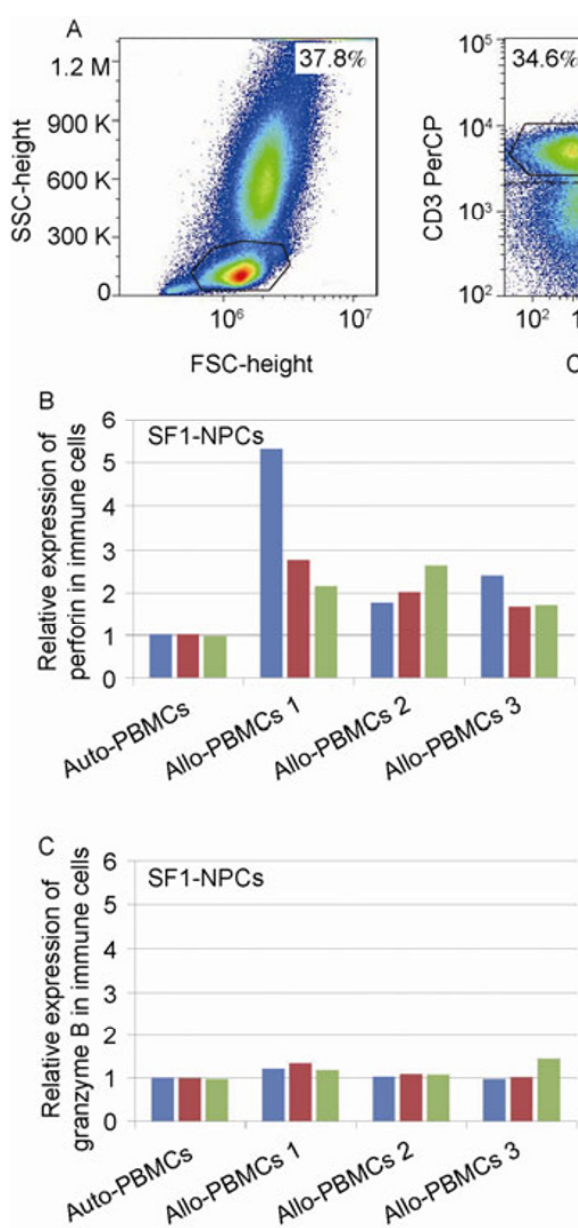
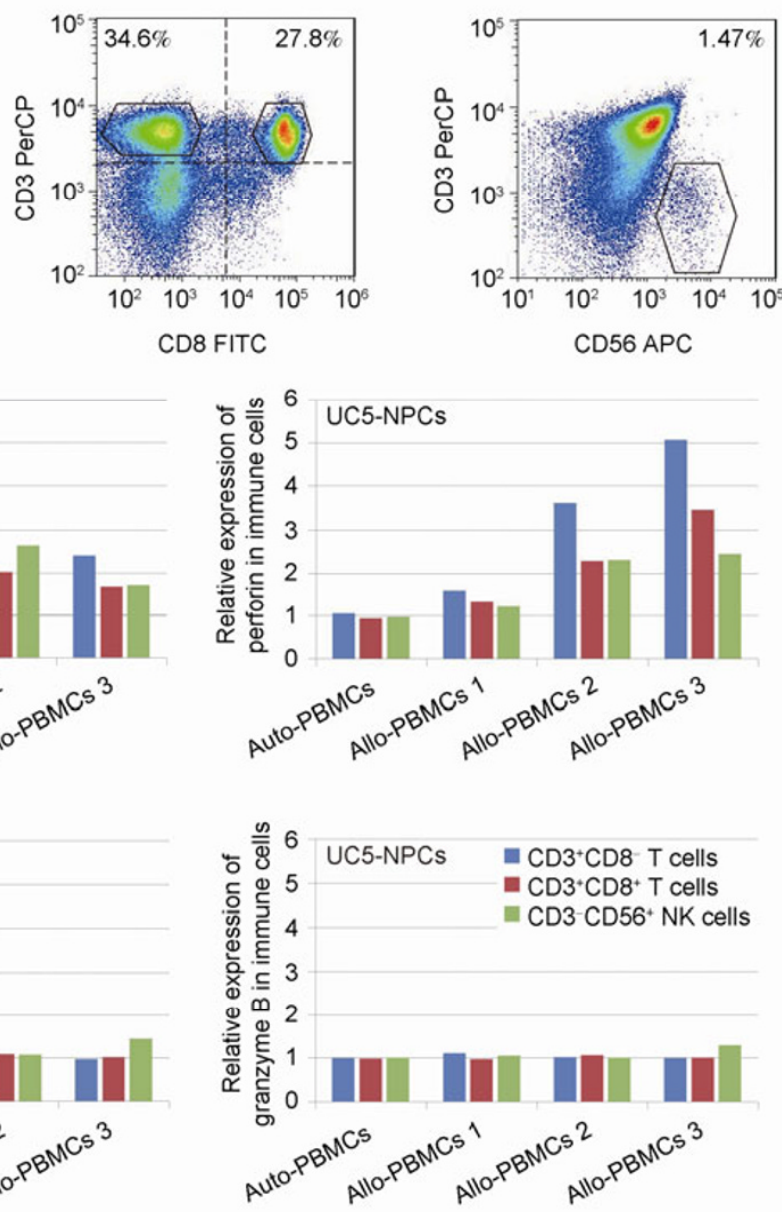

Figure 3 Perforin and granzyme B expressions in a PBMC co-culture system with hiPSC-derived NPC stimulation. A, Analysis of surface phenotypic markers (CD3, CD8, CD56) present on T cells and NK cells. For each cell origin, one strain of autogenous PBMCs and three strains of allogenous PBMCs were individually co-cultured with the relevant NPCs and labeled with appropriate fluorescence-conjugated antibodies. Three obvious cell groups $\left(\mathrm{CD}^{+} \mathrm{CD}^{-} \mathrm{T}\right.$ cells, $\mathrm{CD} 3^{+} \mathrm{CD} 8^{+} \mathrm{T}$ cells and $\mathrm{CD} 3^{-} \mathrm{CD} 56^{+} \mathrm{NK}$ cells) were identified via flow cytometry analysis. $\mathrm{B}$ and $\mathrm{C}$, Analysis of perforin and granzyme B expression in $\mathrm{T}$ cells and NK cells stimulated with SF-NPCs or UC-NPCs. 

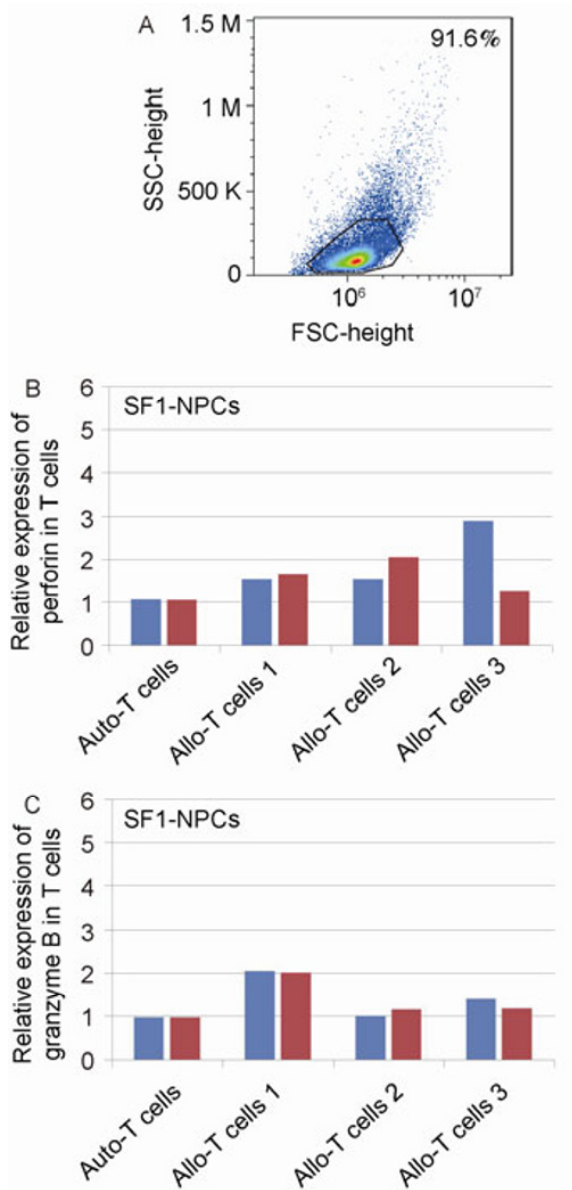
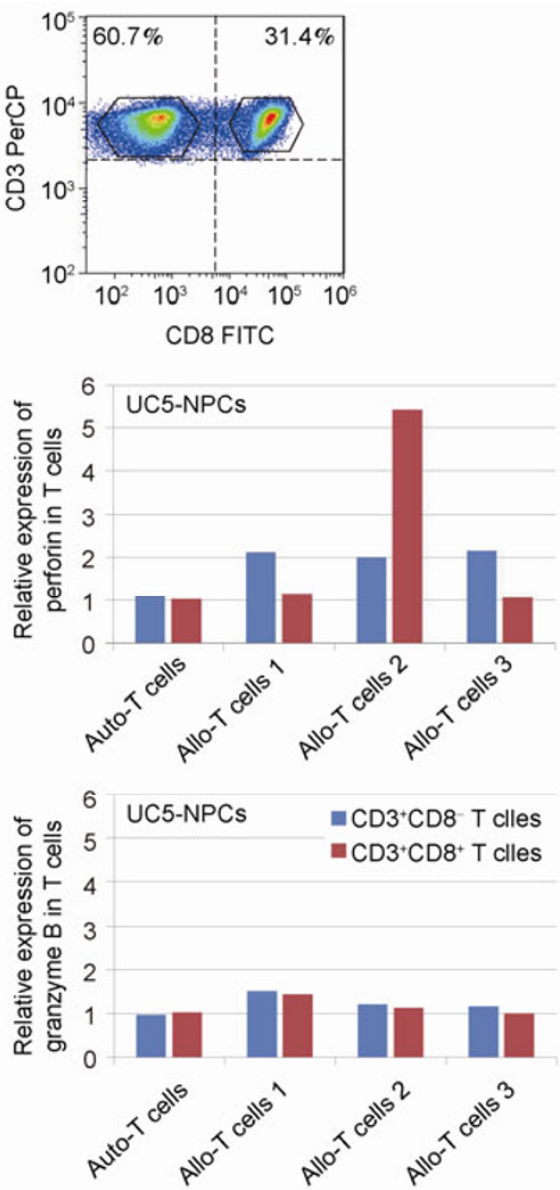

Figure 4 Perforin and granzyme B expressions in a T cell co-culture system with NPC stimulation. A, Analysis of surface phenotypic markers (CD3, CD8) of T cells. For each cell origin, one strain of autogenous T cells and three strains of allogenous T cells were individually co-cultured with the relevant NPCs and labeled with appropriate fluorescence-conjugated antibodies. Two obvious cell groups $\left(\mathrm{CD} 3^{+} \mathrm{CD} 8^{-} \mathrm{T}\right.$ cells and $\mathrm{CD} 3^{+} \mathrm{CD} 8^{+} \mathrm{T}$ cells $)$ were identified by flow cytometry analysis. B and C, Analysis of perforin and granzyme B expression in T cells stimulated with SF-NPCs or UC-NPCs.

ly controversial with the majority of studies based on rodent models [20,22,23]. Here, we have explored the immunogenicity of human iPSC-derived NPCs using a PBMC and T cell co-culture system.

PBMCs include immune effector cells (T cells, B cells and NK cells) and antigen presenting cells (monocytes/macrophages and dendritic cells), which perform a range of different functions during the immune rejection process $[37,38]$. In this study, we examined the proliferation and cytotoxic molecule expression of this mixed cell population following stimulation by somatic cells and NPCs. Relative levels of cellular proliferation and cytotoxic molecule expression were much higher in most allogenous immune cell samples than in autogenous immune cells when stimulated with NPCs. However, the allogeneic T cells present in peripheral blood are mostly in the naive stage, and stimulation by professional antigen presenting cells (APCs) is required for their proliferation. The proliferation observed in allogenous groups (Figure 2) was likely caused by crosspresentation of NPC-derived allogeneic antigens (minor antigens) by APCs such as B cells and monocytes/ macrophages that are included within PBMCs. In other words, the observed proliferation reflects an indirect rather than direct $\mathrm{T}$ cell response. However, to examine direct $\mathrm{T}$ cell simulation, pre-stimulated allo-reactive $\mathrm{T}$ cells rather than PBMCs should be used for the T cell response assay, and a cytokine production assay may prove more sensitive than the proliferation assay employed here. We further investigated the expression of the cytotoxic molecules perforin and granzyme B by several kinds of immune effector cells in a T cell co-culture system (Figure 4). Even though the PBMC or T cell co-culture system imperfectly mimics the in vivo immune response, it is the most reliable human model currently available. Moreover, individual difference is likely to present more difficulties for investigation. Nevertheless, our data showed no large difference in the immunogenicity of NPCs derived from iPSCs created from two different cell sources. SF-iPSCs were generated by transduction with pMX-based retroviruses, while UC-iPSCs were induced with electroporation of oriP/EBNA episomal vectors. The UC-iPSCs in particular are regarded as virus-free and integration-free human iPSC lines, and repre- 
sent a step forward in clinical study of iPSCs. Taken together, our data indicate that these iPSC derivatives are not recognized as allogenous factors by autogenous immune cells and do not initiate a self immune reaction.

Additionally, ZG16 and HORMAD1 have been demonstrated to directly affect the immunogenicity of iPSC derivatives in syngeneic mice [20]. We therefore investigated the expression of ZG16 and HORMAD1 in somatic cells, iPSCs, iPSC-derived teratomas and NPCs of five different cell origins. Limited or no expression of both markers above normal was observed in cells of all origins. ZG16 expression level in each iPSC-derived teratoma was significantly higher, while HORMAD1 was found to remain silenced in the teratomas (Supplemental File 5 in Supporting Information). Therefore, the mechanisms by which ZG16 and HORMAD1 affect immunogenicity may differ. Furthermore, these results suggest that the immunogenicity of iPSCderived teratomas may be affected in the development process, and this may have caused the autogenous T-cell infiltration observed previously [20,21]. However, the silenced expression of ZG16 and HORMAD1 in iPSC-derived NPCs indicates that the differentiation process does not change the immunogenicity of these NPCs and could not activate autogenous lymphocytes. Our current study supports this conclusion (Figures 2-4).

In summary, we have demonstrated that NPCs derived from human iPSCs in vitro are not recognized by autogenous immune cells, while allogenous immune cells were activated by NPCs. These findings suggest that human iPSC-derived NPCs hold potential value for development of further clinical therapies. However, it is impossible to investigate the immunogenicity of human iPSCs and iPSC derivatives in human bodies. Therefore, in addition to in vitro studies, evaluation of the immunogenicity of iPSCs and their derivatives should be further explored in functional humanized animals prior to clinical application.

The authors declare no conflict of interest.

We are grateful to Dr. Qin DaJiang, Dr. Miguel Esteban, Dr. Su Zhong, and Dr. Chen XiaoPing (Guangzhou Institutes of Biomedicine and Health, Chinese Academy of Sciences) for valuable suggestions. This work was supported by the National Basic Research Program of China, Ministry of Science and Technology (2011CB965204, 2012CB966802), the National Natural Science Foundation of China (31000402), the Strategic Priority Research Program of the Chinese Academy of Sciences (XDA01020401, XDA- 01020202), the Ministry of Science and Technology International Technology Cooperation Program (2012DFH30050), the National Science \& Technology Major Special Project on Major New Drug Innovation (2011ZX09102-010-01) and the Development and Technology Innovation for Equipment Functional Development Project of Chinese Academy of Sciences (yg2011082, yg2011083).

1 Thomson JA, Itskovitz-Eldor J, Shapiro SS, Waknitz MA, Swiergiel JJ, Marshall VS, Jones JM. Embryonic stem cell lines derived from human blastocysts. Science, 1998, 282: 1145-1147

2 Odorico JS, Kaufman DS, Thomson JA. Multilineage differentiation from human embryonic stem cell lines. Stem cells, 2001, 19:
193-204

3 Kriks S, Shim JW, Piao J, Ganat YM, Wakeman DR, Xie Z, Carrillo-Reid L, Auyeung G, Antonacci C, Buch A, Yang L, Beal MF, Surmeier DJ, Kordower JH, Tabar V, Studer L. Dopamine neurons derived from human ES cells efficiently engraft in animal models of parkinson's disease. Nature, 2011, 480: 547-551

4 Daadi MM, Grueter BA, Malenka RC, Redmond DE Jr, Steinberg GK. Dopaminergic neurons from midbrain-specified human embryonic stem cell-derived neural stem cells engrafted in a monkey model of parkinson's disease. PLoS ONE, 2012, 7: e41120

5 Lu SJ, Feng Q, Caballero S, Chen Y, Moore MA, Grant MB, Lanza R. Generation of functional hemangioblasts from human embryonic stem cells. Nat Methods, 2007, 4: 501-509

6 Niapour A, Karamali F, Nemati S, Taghipour Z, Mardani M, Nasr-Esfahani MH, Baharvand H. Cotransplantation of human embryonic stem cell-derived neural progenitors and schwann cells in a rat spinal cord contusion injury model elicits a distinct neurogenesis and functional recovery. Cell Transplant, 2012, 21: 827-843

7 Takahashi K, Yamanaka S. Induction of pluripotent stem cells from mouse embryonic and adult fibroblast cultures by defined factors. Cell, 2006, 126: 663-676

8 Takahashi K, Tanabe K, Ohnuki M, Narita M, Ichisaka T, Tomoda K, Yamanaka S. Induction of pluripotent stem cells from adult human fibroblasts by defined factors. Cell, 2007, 131: 861-872

9 Maherali N, Sridharan R, Xie W, Utikal J, Eminli S, Arnold K, Stadtfeld M, Yachechko R, Tchieu J, Jaenisch R, Plath K, Hochedlinger K. Directly reprogrammed fibroblasts show global epigenetic remodeling and widespread tissue contribution. Cell Stem Cell, 2007, 1: 55-70

10 Zhao XY, Li W, Lv Z, Liu L, Tong M, Hai T, Hao J, Guo CL, Ma QW, Wang L, Zeng F, Zhou Q. iPS cells produce viable mice through tetraploid complementation. Nature, 2009, 461: 86-90

11 Spence JR, Mayhew CN, Rankin SA, Kuhar MF, Vallance JE, Tolle K, Hoskins EE, Kalinichenko VV, Wells SI, Zorn AM, Shroyer NF, Wells JM. Directed differentiation of human pluripotent stem cells into intestinal tissue in vitro. Nature, 2011, 470: 105-109

12 Hargus G, Cooper O, Deleidi M, Levy A, Lee K, Marlow E, Yow A, Soldner F, Hockemeyer D, Hallett PJ, Osborn T, Jaenisch R, Isacson O. Differentiated parkinson patient-derived induced pluripotent stem cells grow in the adult rodent brain and reduce motor asymmetry in parkinsonian rats. Proc Natl Acad Sci USA, 2010, 107: 15921-15926

13 Wang Y, Zheng CG, Jiang Y, Zhang J, Chen J, Yao C, Zhao Q, Liu S, Chen K, Du J, Yang Z, Gao S. Genetic correction of beta-thalassemia patient-specific iPS cells and its use in improving hemoglobin production in irradiated SCID mice. Cell Res, 2012, 22: 637-648

14 Merkle FT, Eggan K. Modeling human disease with pluripotent stem cells: from genome association to function. Cell Stem Cell, 2013, 12: 656-668

15 Reinhardt P, Schmid B, Burbulla LF, Schöndorf DC, Wagner L, Glatza M, Höing S, Hargus G, Heck SA, Dhingra A, Wu G, Müller S, Brockmann K, Kluba T, Maisel M, Krüger R, Berg D, Tsytsyura Y, Thiel CS, Psathaki OE, Klingauf J, Kuhlmann T, Klewin M, Müller H, Gasser T, Schöler HR, Sterneckert J. Genetic correction of a LRRK2 mutation in human iPSCs links parkinsonian neurodegeneration to ERK-dependent changes in gene expression. Cell Stem Cell, 2013, 12: 354-367

16 Sebastiano V, Maeder ML, Angstman JF, Haddad B, Khayter C, Yeo DT, Goodwin MJ, Hawkins JS, Ramirez CL, Batista LF, Artandi SE, Wernig M, Joung JK. In situ genetic correction of the sickle cell anemia mutation in human induced pluripotent stem cells using engineered zinc finger nucleases. Stem Cells, 2011, 29: 1717-1726

17 Sancho-Bru P, Roelandt P, Narain N, Pauwelyn K, Notelaers T, Shimizu T, Ott M, Verfaillie C. Directed differentiation of murine-induced pluripotent stem cells to functional hepatocyte-like cells. J Hepatol, 2011, 54: 98-107

18 Si-Tayeb K, Noto FK, Nagaoka M, Li J, Battle MA, Duris C, North PE, Dalton S, Duncan SA. Highly efficient generation of human hepatocyte-like cells from induced pluripotent stem cells. Hepatology, 2010, 51: 297-305 
19 Fairchild PJ. The challenge of immunogenicity in the quest for induced pluripotency. Nat Rev Immunol, 2010, 10: 868-875

20 Zhao T, Zhang ZN, Rong Z, Xu Y. Immunogenicity of induced pluripotent stem cells. Nature, 2011, 474: 212-215

21 Okita K, Nagata N, Yamanaka S. Immunogenicity of induced pluripotent stem cells. Circulat Res, 2011, 109: 720-721

22 Araki R, Uda M, Hoki Y, Sunayama M, Nakamura M, Ando S, Sugiura M, Ideno H, Shimada A, Nifuji A, Abe M. Negligible immunogenicity of terminally differentiated cells derived from induced pluripotent or embryonic stem cells. Nature, 2013, 494: $100-104$

23 Guha P, Morgan JW, Mostoslavsky G, Rodrigues NP, Boyd AS. Lack of immune response to differentiated cells derived from syngeneic induced pluripotent stem cells. Cell Stem Cell, 2013, 12: $407-412$

24 Inoue $\mathrm{CN}$, Sunagawa N, Morimoto T, Ohnuma S, Katsushima F, Nishio T, Kondo Y, Iinuma K. Reconstruction of tubular structures in three-dimensional collagen gel culture using proximal tubular epithelial cells voided in human urine. In Vitro Cell Dev Biol Anim, 2003, 39: 364-367

25 Zhou T, Benda C, Duzinger S, Huang Y, Li X, Li Y, Guo X, Cao G, Chen S, Hao L, Chan YC, Ng KM, Ho JC, Wieser M, Wu J, Redl H, Tse HF, Grillari J, GrillariVoglauer R, Pei D, Esteban MA. Generation of induced pluripotent stem cells from urine. J Am Soc Nephrol, 2011, 22: 1221-1228

26 Cai J, Li W, Su H, Qin D, Yang J, Zhu F, Xu J, He W, Guo X, Labuda K, Peterbauer A, Wolbank S, Zhong M, Li Z, Wu W, So KF, Redl H, Zeng L, Esteban MA, Pei D. Generation of human induced pluripotent stem cells from umbilical cord matrix and amniotic membrane mesenchymal cells. J Biol Chem, 2010, 285: 1122711234

27 Yu J, Hu K, Smuga-Otto K, Tian S, Stewart R, Slukvin II, Thomson JA. Human induced pluripotent stem cells free of vector and transgene sequences. Science, 2009, 324: 797-801

28 Liao B, Bao X, Liu L, Feng S, Zovoilis A, Liu W, Xue Y, Cai J, Guo X, Qin B, Zhang R, Wu J, Lai L, Teng M, Niu L, Zhang B, Esteban MA, Pei D. microRNA cluster 302-367 enhances somatic cell reprogramming by accelerating a mesenchymal-to-epithelial transition. J Biol Chem, 2011, 286: 17359-17364

29 Almici C, Carlo-Stella C, Mangoni L, Garau D, Cottafavi L, Ventura A, Armanetti M, Wagner JE, Rizzoli V. Density separation of umbilical cord blood and recovery of hemopoietic progenitor cells: implications for cord blood banking. Stem Cells, 1995, 13: 533-540

30 Liu P, Chen S, Li X, Qin L, Huang K, Wang L, Huang W, Li S, Jia B, Zhong M, Pan G, Cai J, Pei D. Low immunogenicity of neural progenitor cells differentiated from induced pluripotent stem cells derived from less immunogenic somatic cells. PLoS ONE, 2013, 8: e69617

31 Wagner JE, Collins D, Fuller S, Schain LR, Berson AE, Almici C, Hall MA, Chen KE, Okarma TB, Lebkowski JS. Isolation of small, primitive human hematopoietic stem cells: distribution of cell surface cytokine receptors and growth in SCID-Hu mice. Blood, 1995, 86: 512-523

32 Farowski F, Cornely OA, Vehreschild JJ, Bauer T, Hartmann P, Steinbach A, Vehreschild MJ, Scheid C, Müller C. Intracellular concentrations of micafungin in different cellular compartments of the peripheral blood. Int J Antimicrob Agents, 2012, 39: 228-231

33 Prasanna SJ, Gopalakrishnan D, Shankar SR, Vasandan AB. Pro-inflammatory cytokines, IFNgamma and TNFalpha, influence immune properties of human bone marrow and Wharton jelly mesenchymal stem cells differentially. PLoS ONE, 2010, 5: e9016

34 Jangpatarapongsa K, Xia H, Fang Q, Hu K, Yuan Y, Peng M, Gao Q, Sattabongkot J, Cui L, Li B, Udomsangpetch R. Immunity to malaria in plasmodium vivax infection: a study in central China. PLoS ONE, 2012, 7: e45971

35 Yang J, Cai J, Zhang Y, Wang X, Li W, Xu J, Li F, Guo X, Deng K, Zhong M, Chen Y, Lai L, Pei D, Esteban MA. Induced pluripotent stem cells can be used to model the genomic imprinting disorder Prader-Willi syndrome. J Biol Chem, 2010, 285: 40303-40311

36 Kim DS, Lee JS, Leem JW, Huh YJ, Kim JY, Kim HS, Park IH, Daley GQ, Hwang DY, Kim DW. Robust enhancement of neural differentiation from human ES and iPS cells regardless of their innate difference in differentiation propensity. Stem Cell Rev, 2010, 6: 270-281

37 Duffy MM, Ritter T, Ceredig R, Griffin MD. Mesenchymal stem cell effects on T-cell effector pathways. Stem cell research \& therapy, 2011, 2: 34

38 Moretta L, Locatelli F, Pende D, Marcenaro E, Mingari MC, Moretta A. Killer Ig-like receptor-mediated control of natural killer cell alloreactivity in haploidentical hematopoietic stem cell transplantation. Blood, 2011, 117: 764-771

Open Access This article is distributed under the terms of the Creative Commons Attribution License which permits any use, distribution, and reproduction in any medium, provided the original author(s) and source are credited.

\section{Supporting Information}

Supplemental File 1 Percentage of perforin expression in various immune effector cells in the PBMC co-culture system.

Supplemental File 2 Percentage of granzyme B expression in various immune effector cells in the PBMC co-culture system.

Supplemental File 3 Percentage of perforin expression in the immune effector cells in the T cell co-culture system.

Supplemental File 4 Percentage of granzyme B expression in the immune effector cells in the T cell co-culture system.

Supplemental File 5 Expression of ZG16 and HORMAD1 determined via qPCR. Similar results were obtained in three independent experiments. Results are expressed as mean \pm SEM.

The supporting information is available online at life.scichina.com and link.springer.com. The supporting materials are published as submitted, without typesetting or editing. The responsibility for scientific accuracy and content remains entirely with the authors. 